\title{
Annexe
}

\section{Liste des laboratoires et organismes}

Les listes ci-dessous ne sont pas exhaustives, elles ne concernent que les laboratoires publics et organismes cités par les auteurs qui ont contribué à la rédaction des articles de ce Livre blanc.

Il s'agit de listes élaborées à la date d'édition. Pour obtenir une liste plus récente, le lecteur est invité à se connecter au site de l'AFM www.afm.asso.fr, espace adhérents.

\begin{tabular}{|l|l|l|l|}
\hline \multicolumn{1}{|c|}{ Laboratoire } & \multicolumn{1}{|c|}{ Organisme } & \multicolumn{1}{|c|}{ Ville } & \multicolumn{1}{c|}{$\begin{array}{c}\text { Chapitres } \\
\text { Livlanc }\end{array}$} \\
\hline B2OA - UMR 7052- & Université Paris 7 & Paris & 3.9 \\
\hline $\begin{array}{l}\text { Biomécanique et } \\
\text { bio-ingénierie - UMR 7338 }\end{array}$ & UTC & Compiègne & 3.9 \\
\hline CEAT & $\begin{array}{l}\text { Université de } \\
\text { Poitiers ENSMA }\end{array}$ & Poitiers & $3.5,3.10$ \\
\hline CEMEF - UMR 7635 & $\begin{array}{l}\text { CEMEF - Mines } \\
\text { ParisTech - } \\
\text { École des mines }\end{array}$ & Sophia Antipolis & $\begin{array}{l}3.7,3.14,3.15, \\
4.2\end{array}$ \\
\hline $\begin{array}{l}\text { Centre des matériaux } \\
\text { - UMR 7633 }\end{array}$ & Mines ParisTech & Évry & $\begin{array}{l}3.4,3.14,3.15, \\
4.2\end{array}$ \\
\hline CGS & Mines ParisTech & Paris & 4.1 \\
\hline CIRIMAT - UMR 5085 & $\begin{array}{l}\text { Université de } \\
\text { Toulouse III }\end{array}$ & Toulouse & 4.2 \\
\hline DIPI & ENISE & Saint-Étienne & 4.2 \\
\hline DYNFLUID & ENSAM, CER & Paris & 3.11 \\
\hline EMC2 - UPR 288 & École centrale & Paris & $3.11,3.13$ \\
\hline ENSMP & Mines ParisTech & Paris & 3.6 \\
\hline ERPI & Université de & Nancy & 4.1 \\
\hline FAST - UMR 7608 & $\begin{array}{l}\text { Eorraine } \\
\text { polytechnique }\end{array}$ & Palaiseau & 3.2 \\
\hline GEM - UMR 6183 & École centrale & Nantes & $\begin{array}{l}3.4,3.6,3.14, \\
3.15\end{array}$ \\
\hline
\end{tabular}




\begin{tabular}{|c|c|c|c|}
\hline Laboratoire & Organisme & Ville & $\begin{array}{l}\text { Chapitres } \\
\text { Livre blanc }\end{array}$ \\
\hline $\begin{array}{l}\text { Génie des systèmes } \\
\text { mécaniques }\end{array}$ & UTC & Compiègne & 3.17 \\
\hline G-SCOP - UMR 5272 & INPG & Grenoble & $4.1,4.2,4.3$ \\
\hline I2M - UMR 5295 & $\begin{array}{l}\text { Université } \\
\text { Bordeaux }\end{array}$ & Bordeaux & $\begin{array}{l}3.4,3.10,3.12, \\
3.15,3.16,4.1, \\
4.2\end{array}$ \\
\hline I3M - UMR 5149 & $\begin{array}{l}\text { Université } \\
\text { Montpellier II }\end{array}$ & Montpellier & 3.11 \\
\hline ICB-LTM - UMR 6303 & $\begin{array}{l}\text { Université de } \\
\text { Bourgogne }\end{array}$ & Le Creusot & 4.2 \\
\hline ICMMO & $\begin{array}{l}\text { Université } \\
\text { Paris-Sud }\end{array}$ & Orsay & 4.2 \\
\hline IMFT - UMR 5502 & IMFT & Toulouse & 3.1 \\
\hline IMP - UMR 5223 & INSA & Lyon & 4.2 \\
\hline $\begin{array}{l}\text { Institut Clément-Ader } \\
\text { - EA } 814\end{array}$ & $\begin{array}{l}\text { Université } \\
\text { Toulouse III }\end{array}$ & Toulouse & $3.4,3.6,4.2$ \\
\hline $\begin{array}{l}\text { Institut des sciences du mou- } \\
\text { vement (UMR 7287) }\end{array}$ & $\begin{array}{l}\text { Aix-Marseille } \\
\text { université }\end{array}$ & Marseille & 3.9 \\
\hline $\begin{array}{l}\text { Institut FEMTO-ST } \\
\text { - UMR } 6174\end{array}$ & $\begin{array}{l}\text { Université de } \\
\text { Franche-Comté / } \\
\text { ENSMM / UTBM }\end{array}$ & Besançon & $3.15,4.1,4.2$ \\
\hline $\begin{array}{l}\text { Institut Jean-Lamour (IJL) - } \\
\text { UMR } 7198\end{array}$ & $\begin{array}{l}\text { Université de } \\
\text { Lorraine }\end{array}$ & Nancy & 3.15 \\
\hline $\begin{array}{l}\text { Institut Jean-Le-Rond- } \\
\text { d'Alembert (IJRA) - } \\
\text { UMR } 6174 \text { et } 7130\end{array}$ & $\begin{array}{l}\text { Université Pierre- } \\
\text { et-Marie-Curie }\end{array}$ & Paris & $3.1,3.11$ \\
\hline $\begin{array}{l}\text { Institut Navier ENSPC - } \\
\text { UMR } 8205\end{array}$ & $\begin{array}{l}\text { École des ponts } \\
\text { ParisTech }\end{array}$ & Marne-la-Vallee & $3.2,3.6,4.2$ \\
\hline Institut Pascal - UMR 6602 & $\begin{array}{l}\text { Université Blaise- } \\
\text { Pascal - IFMA }\end{array}$ & Aubière & $3.4,4.1,4.2,4.3$ \\
\hline Institut Pprime - UPR 3346 & $\begin{array}{l}\text { ENSMA } \\
\text { Université } \\
\text { de Poitiers }\end{array}$ & $\begin{array}{l}\text { Futuroscope } \\
\text { Chasseneuil-du- } \\
\text { Poitou }\end{array}$ & $3.6,3.7,3.11,4.2$ \\
\hline IPR - UMR 6265 & $\begin{array}{l}\text { Université de } \\
\text { Rennes }\end{array}$ & Rennes & 3.2 \\
\hline IRCCyN - UMR 6597 & École centrale & Nantes & $4.1,4.2,4.3$ \\
\hline IRPHE - UMR 7342 & $\begin{array}{l}\text { Université } \\
\text { d'Aix-Marseille }\end{array}$ & Marseille & $3.1,4.2$ \\
\hline IUSTI - UMR 6595 & $\begin{array}{l}\text { Université de } \\
\text { Marseille }\end{array}$ & Marseille & 3.1 \\
\hline LABOMAP - EA 3633 & ENSAM & Cluny & 4.2 \\
\hline
\end{tabular}




\begin{tabular}{|c|c|c|c|}
\hline Laboratoire & Organisme & Ville & $\begin{array}{l}\text { Chapitres } \\
\text { Livre blanc }\end{array}$ \\
\hline $\begin{array}{l}\text { Laboratoire de bioméca- } \\
\text { nique - EA } 4494\end{array}$ & ENSAM & Paris & 3.9 \\
\hline LADHYX - UMR 7646 & $\begin{array}{l}\text { École } \\
\text { polytechnique }\end{array}$ & Palaiseau & 3.1 \\
\hline LAMCOS - UMR 5259 & INSA de Lyon & Villeurbanne & $\begin{array}{l}3.7,3.9,3.11 \\
3.14,3.15,4.2\end{array}$ \\
\hline LAMIH - UMR8201 & $\begin{array}{l}\text { Université de } \\
\text { Valenciennes }\end{array}$ & Valenciennes & $3.4,3.9$ \\
\hline LAMPA & ENSAM & Angers & 4.2 \\
\hline LAMSID - UMR 8193 & CNRS-EDF & Clamart & $3.4,3.15$ \\
\hline LASIE - UMR 7356 & $\begin{array}{l}\text { LASIE - } \\
\text { Université } \\
\text { de La Rochelle }\end{array}$ & La Rochelle & 3.1 \\
\hline LASMIS - UMR 2848 & $\begin{array}{l}\text { Université } \\
\text { d technologie } \\
\text { de Troyes (UTT) }\end{array}$ & Troyes & $3.15,4.1,4.2$ \\
\hline LAUM - UMR 6613 & $\begin{array}{l}\text { Université } \\
\text { du Maine }\end{array}$ & Le Mans & 3.11 \\
\hline LBA - UMR T24 & $\begin{array}{l}\text { Université } \\
\text { d'Aix-Marseille }\end{array}$ & Marseille & 3.9 \\
\hline LBMS & ENSTA Bretagne & Brest & 4.2 \\
\hline LCFC - EA 4495 & ENSAM & Metz & $3.4,4.1,4.2,4.3$ \\
\hline LCPI - EA 3927 & ENSAM & Paris & $4.1,4.2$ \\
\hline LCPO - UMR 5629 & $\begin{array}{l}\text { Université } \\
\text { de Bordeaux }\end{array}$ & Pessac & 4.2 \\
\hline LEGI - UMR 5519 & LEGI - CNRS & Grenoble & 3.1 \\
\hline LEM3 -UMR 7239 & $\begin{array}{l}\text { Université } \\
\text { de Lorraine }\end{array}$ & Metz & $3.14,3.15,4.2$ \\
\hline LEMTA - UMR 7563 & $\begin{array}{l}\text { Université } \\
\text { de Lorraine }\end{array}$ & $\begin{array}{l}\text { Vandœuvre-lès- } \\
\text { Nancy }\end{array}$ & $3.9,4.2$ \\
\hline LEPS/STOICA - UMR 5600 & INSA & Lyon & $4.1,4.2$ \\
\hline LERMPS - EA7274 & UTBM & Belfort & 4.2 \\
\hline LFM - EA 4362 & $\begin{array}{l}\text { Université } \\
\text { de Lorraine }\end{array}$ & Metz & 3.9 \\
\hline LGECO - EA 3938 & INSA & Strasbourg & $4.1,4.2$ \\
\hline LGI - EA 2606 & École centrale & Paris & $4.1,4.2$ \\
\hline LGP - EA 1905 & ENIT & Tarbes & 4.2 \\
\hline LHEEA - UMR 6598 & École centrale & Nantes & 3.10 \\
\hline LIMATB & $\begin{array}{l}\text { Université } \\
\text { Bretagne-Sud }\end{array}$ & Lorient & 3.15 \\
\hline
\end{tabular}




\begin{tabular}{|c|c|c|c|}
\hline Laboratoire & Organisme & Ville & $\begin{array}{l}\text { Chapitres } \\
\text { Livre blanc }\end{array}$ \\
\hline LIMSI - UPR 3251 & $\begin{array}{l}\text { Université } \\
\text { Paris-Sud }\end{array}$ & Orsay & 3.2 \\
\hline LIPHY - UMR 5588 & $\begin{array}{l}\text { Université } \\
\text { Joseph-Fourier } \\
\text { - INPG }\end{array}$ & $\begin{array}{l}\text { Saint-Martin- } \\
\text { d'Hères }\end{array}$ & 3.2 \\
\hline LISMMA - EA 2336 & Supméca & Saint-Ouen & $\begin{array}{l}3.7,3.11,3.17, \\
4.1,4.2\end{array}$ \\
\hline LM3 - UMR 7239 & $\begin{array}{l}\text { Université } \\
\text { de Metz }\end{array}$ & Metz & 3.2 \\
\hline LMA - UPR 7051 & $\begin{array}{l}\text { Université } \\
\text { de Marseille }\end{array}$ & Marseille & $\begin{array}{l}3.2,3.4,3.6,3.11 \text {, } \\
3.14\end{array}$ \\
\hline LMBC, UMR_T 9406 & Université Lyon 1 & Lyon & 3.9 \\
\hline LMFA - UMR 5509 & $\begin{array}{l}\text { École centrale } \\
\text { de Lyon }\end{array}$ & Écully & $3.1,3.11$ \\
\hline LMGC & IUT & Nîmes & 3.15 \\
\hline LMGC - UMR 5508 & $\begin{array}{l}\text { Université } \\
\text { Montpellier } 2\end{array}$ & Montpellier & $3.9,3.14$ \\
\hline LML - UMR 8107 & Cité scientifique & Villeneuve-d'Ascq & $3.1,3.14$ \\
\hline LMR - EA 2640 & $\begin{array}{l}\text { Université } \\
\text { François-Rabelais } \\
\text { - Tours }\end{array}$ & Tours & 3.11 \\
\hline LMSME - UMR 8208 & $\begin{array}{l}\text { Université } \\
\text { Marne-la-Vallée }\end{array}$ & Marne-la-Vallée & 3.4 \\
\hline LMSSM - UMR 8579 & École centrale & Chatenay-Malabry & 3.4 \\
\hline LMT - UMR 8535 & ENS Cachan & Cachan & $\begin{array}{l}3.6,3.14,3.15, \\
4.2\end{array}$ \\
\hline LOFIMS & INSA & Rouen & 3.4 \\
\hline LOMA - UMR 5798 & $\begin{array}{l}\text { Université } \\
\text { de Bordeaux }\end{array}$ & Bordeaux & $3.2,4.2$ \\
\hline LP - UMR 5672 & ENS Lyon & Lyon & 3.2 \\
\hline LPIM - UMR 7525 & $\begin{array}{l}\text { Université } \\
\text { de Haute-Alsace }\end{array}$ & Mulhouse & 4.2 \\
\hline LPS - UMR 8550 & ENS UPMC & Paris & 3.2 \\
\hline LSIS - UMR 7296 & ENSAM & Aix-en-Provence & $4.1,4.3$ \\
\hline LTDS - UMR 5513 & EC Lyon & Écully & $\begin{array}{l}3.7,3.11,3.14, \\
3.15,4.2\end{array}$ \\
\hline LURPA - EA 1385 & ENS & Cachan & $4.1,4.2,4.3$ \\
\hline LVA - EA 677 & INSA & Lyon & 3.11 \\
\hline M2P2 - UMR 7340 & IMT & Marseille & 3.1 \\
\hline M3M - EA 3318 & UTBM & Belfort & $4.1,4.2$ \\
\hline
\end{tabular}




\begin{tabular}{|l|l|l|l|}
\hline \multicolumn{1}{|c|}{ Laboratoire } & \multicolumn{1}{|c|}{ Organisme } & \multicolumn{1}{|c|}{$\begin{array}{c}\text { Chille } \\
\text { Livre blanc }\end{array}$} \\
\hline MATEIS - UMR 5510 & INSA & Lyon & 3.6 \\
\hline MSC - UMR 7057 & Université Paris 7 & Paris & 3.2 \\
\hline MSME - UPEC UMR 8208 & $\begin{array}{l}\text { Université de } \\
\text { Marne-la-Vallée }\end{array}$ & Créteil & $3.4,3.9,3.11$ \\
\hline PIM - UMR 8006 & ENSAM & Paris & 4.2 \\
\hline PIMM - UMR 8006 & $\begin{array}{l}\text { Arts et Métiers } \\
\text { ParisTech }\end{array}$ & Paris & $3.6,3.15$ \\
\hline PMMH - UMR 7636 & ESPCI ParisTech & Paris & 3.1 \\
\hline POEMS - UMR 7231 & ENSTA & Paris & 3.11 \\
\hline PPMD/SIM - UMR 7615 & ESPCI ParisTech & Paris & $3.2,4.2$ \\
\hline Roberval - UMR 7337 & UTC & Compiègne & $\begin{array}{l}3.4,3.11,3.14, \\
3.15,4.1,4.2\end{array}$ \\
\hline SIMaP - UMR 5266 & INPG & Grenoble & 3.15 \\
\hline SYMME - UMR 5271 & $\begin{array}{l}\text { Université } \\
\text { de Savoie }\end{array}$ & Annecy & 3.17 \\
\hline TEMPO - EA 4542 & $\begin{array}{l}\text { Université de } \\
\text { Valenciennes }\end{array}$ & Valenciennes & $3.15,4.2$ \\
\hline UME - UMR 7652 & ENSTA ParisTech & Paris & $3.2,3.11,3.15$ \\
\hline URCA & $\begin{array}{l}\text { Université } \\
\text { de Reims }\end{array}$ & Reims & $3.15,4.2$ \\
\hline X-LMS - UMR 7649 & $\begin{array}{l}\text { École des } \\
\text { mines/École } \\
\text { polytechnique }\end{array}$ & Palaiseau & $3.2,3.14$ \\
\hline
\end{tabular}

Liste des organismes de recherche publics et centres techniques référencés dans les articles :

BRGM, CEA, CERFACS, CETIAT, CETIM, CNRS, CSTB, IFPEN, IFSTTAR, INSIS, Institut de soudure, IFREMER, IREPA Laser, IRSTEA, ONERA, PEP. 
